\title{
Combining X-Rays and Neutrons to Shed Light in the Conformational Changes in Aminoacids
}

\author{
Margareth KKD Franco*1 and FabianoYokaichiya ${ }^{2}$ \\ ${ }^{1}$ Instituto de Pesquisas Energéticase Nucleares - IPEN, Brazil \\ ${ }^{2}$ Helmholtz-Zentrum-Berlin, Germany
}

Received: 盋 August 17, 2018; Published: 眥 August 27, 2018

*Corresponding author: Margareth KKD Franco, Instituto de Pesquisas Energéticase Nucleares - IPEN, Brazil

\begin{abstract}
Amino acids are small organic molecules having both amino and carboxylic acid groups bound to the $\alpha$-carbon, with a general formula NH2$\mathrm{CH}(\mathrm{R})-\mathrm{COOH}$. Their side chains, R, can vary - saml our bulky, hidrophobic our hydrophilic, polar, charged or neutral. If the $\alpha$-carbon is bound to four different chemical groups, the amino acid is chiral and two isomers exist labeled with L our D. Furthermore, the parameters characterizing the motions of amino acids in crystal can be comparable with those describing this dynamics in biopolymers. Therefore, possessing information on the strusture and dynamics of single amino acids is important in biochemistry, biophysics, medicine and pharmacy. The structure and dynamics of crystalline amino acids can be followed in a wide temperature and pressure range by diffraction and various spectroscopic techniques and the results can be interpreted, giving the characteristic times of selected motions and the values of related energy barriers.
\end{abstract}

Keywords: X-Ray; Neutron; Amino Acid; Structure-Property

\section{Introduction}

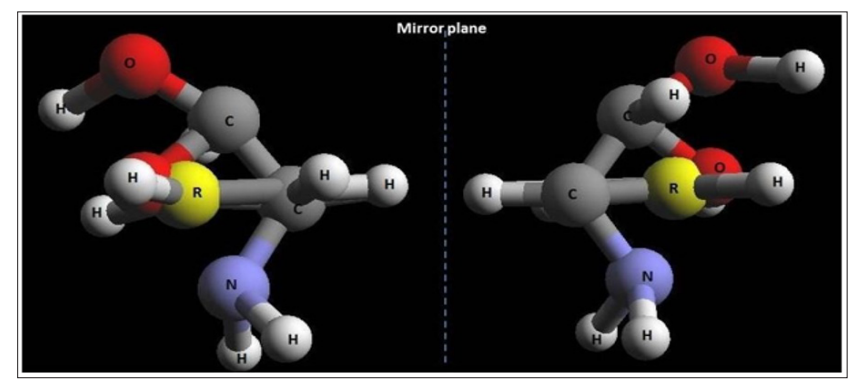

Figure 1: D-amino acid and L-amino acid.

L-methionine (Figure 1) is an essential amino acid important in the methylation process playing a key role in the immune system affected by HIV [1] and other diseases (like Alzheimer and Parkinson [2]) . However very few studies crystalline L- methionine have been reported in the literature [3] thus very little is known about its physical properties. The aim of this study is to investigate the stucture-property relations in L- methionine as a function temperature, because of according to recent experiments, including Raman spectroscopy, thermal analysis, nêutron diffraction and inelastic neutron scattering, changes in the conformational states of L-methionine induce a variety of structural arrangements between 220 and $340 \mathrm{~K}$. Here we present the first results from X-ray Synchrotron(LNLS-XPD) and neutron diffraction (IPEN) which combined to the previous results confirm that subtle structural changes in L- Methionine lead to a rich phase diagram in this important amino acid

\section{Materials and Methods}

The L-methionine powder samples measured, using neutron and X-ray diffraction, were acquire from Sigma-Aldrich and no further treatment (like purification) were performed.

\section{Neutron Powder Diffractometer (NPD) Aurora-IPEN}

The neutron powder diffractometer used in the study is Aurora, a high-resolution powder diffractometer, placed on the research reactor IEA-R1, located at the Instituto de Pesquisas Energéticas e Nucleares (IPEN-CNEN, Sao Paulo) [4]. Aurora diffractometer set up is designed with a position sensitive detector (PSD) and a silicon double-focusing single crystal monochromator. In order to perform the measurement in the L-methionine powder samples, an adjusted value for the wavelength of 1.399 Å was used, improving the resolution in the q-range measured. The experiment was performed at room temperature, and a vanadium container was used to hold the sample.

\section{X-ray powder diffractometer (XPD)}

TheX-ray powder diffractometer to investigate the L-methionine systems for different temperatures was performed at D10B-XPD [5] in the Brazilian Synchrotron Laboratory (LNLS) using a 4+2 circle Huber diffractometer in a high-resolution mode, with a Ge 111 
analyser crystal, using a wavelength of $1.653 \AA$ A. The high-resolution mode was chosen in order to get a better resolution in the final data to facilitate the Rietveld refinement. The data were obtained using a He closed-cycle cryostat for three temperatures: $100 \mathrm{~K}, 200 \mathrm{~K}$ and $300 \mathrm{~K}$, in a $\theta-2 \theta$ geometry (Bragg-Bretano configuration), with a flat plane sample holder, over a $2 \theta$ range of 10 to $100^{\circ}$. The advantage of measuring with synchrotron source when compared with a conventional X-ray source is the photon flux and the resolution. The undesirable disadvantage, that was controlled in this experiment, is the radiation damage in biological materials.

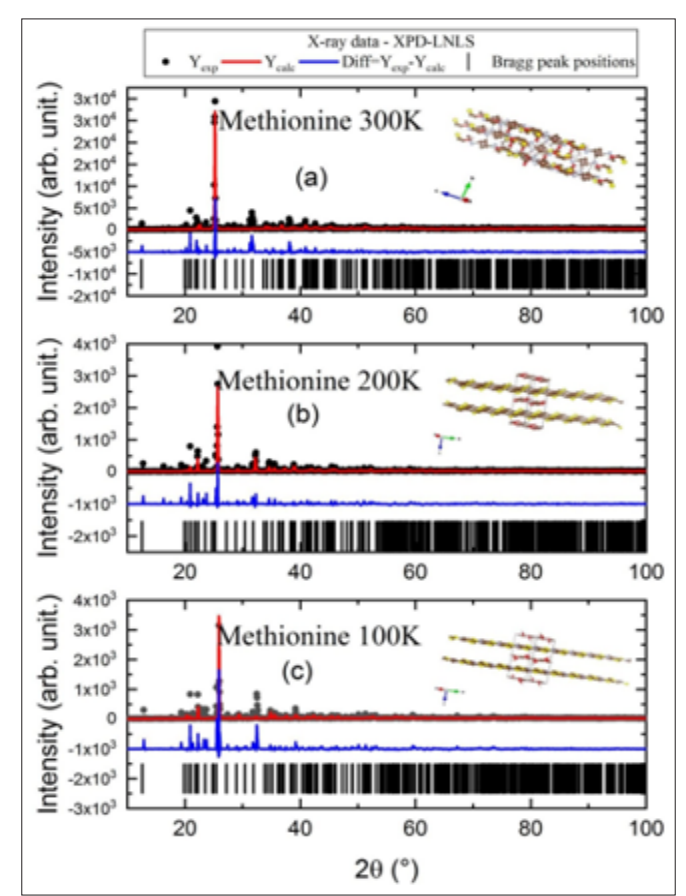

Figure 2: Refinement, using software TOPAS, of the X-ray powder diffraction data measured at XPD-LNLS for $100 \mathrm{~K}, 200 \mathrm{~K}$ and $300 \mathrm{~K}$.

\section{Rietveld Refinement}

In this study, for both diffraction experiments, a Rietveld analysis [6] was performed for the powder diffraction data using programs FULLPROF [7] and TOPAS [8]. Frequently, the refinements plots show both experimental and calculated data, as well as the difference between them displayed below as a single diagram as we can observe in the Figure 2. In addition, the Bragg peak positions are also depicted at the Figures 3.

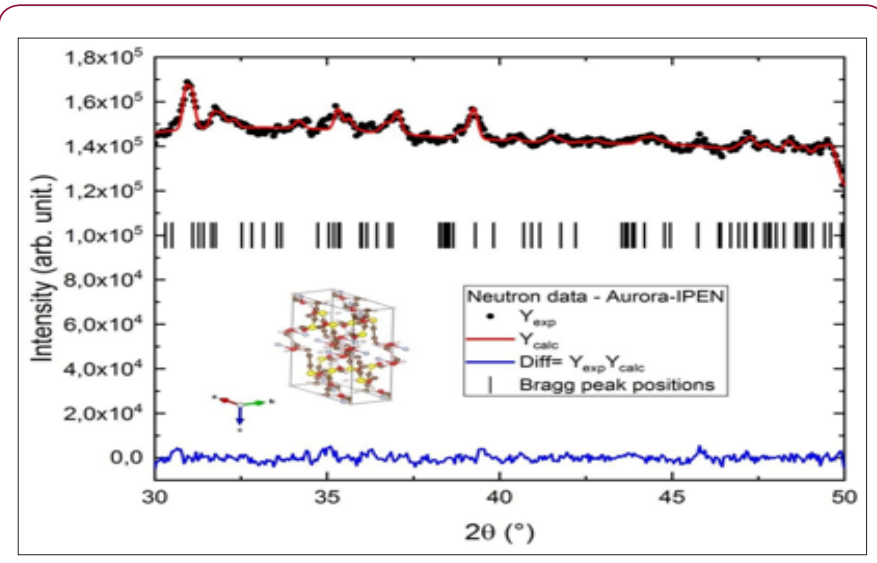

Figure 3: Refinement, using software TOPAS, of the neutron powder diffraction data measured at AuroraIPEN at $300 \mathrm{~K}$.

\section{Results and Discussions}

In order to show the importance of the application of techniques like Synchrotron X- ray and neutron powder diffraction in the investigation of properties of important biological materials, we study the behavior of the structural changings as a function of temperature on L-methionine for three selected temperatures: 300 $\mathrm{K}$ (neutron and X-ray combined), 200K (X-ray) and 100K (X-ray). The major idea for the study using neutron and X-ray powder diffraction techniques for $300 \mathrm{~K}$, is to combined both experiments to get a better refinement results. The results from the combined refinement (neutron and X-ray) is shown in the Figure 4 and in the Table 1, where we displayed the lattice parameters $(a, b, c)$ and the beta angle for the monoclic $(\mathrm{C} 2 / \mathrm{c})$ structure of L-methionine. To obtain the lattice parameter for 200 and $100 \mathrm{~K}$, our start point was using the $300 \mathrm{~K}$ results and the results are also displayed in the Figure 4 and Table 1.

Table 1: List of lattice parameter $(a, b, c)$, beta angle and volume for L-methionine for 3 temperatures.

\begin{tabular}{|c|c|c|c|}
\hline & $\mathbf{1 0 0 K}$ & $\mathbf{2 0 0 K}$ & 300K \\
\hline $\mathrm{a}(\AA)$ & $9.502(6)$ & $9.605(7)$ & $9.649(1)$ \\
\hline $\mathrm{b}(\AA)$ & $5.230(5)$ & $5.178(8)$ & $5.207(6)$ \\
\hline $\mathrm{c}(\AA)$ & $14.971(8)$ & $15.138(8)$ & $15.372(8)$ \\
\hline$\beta\left({ }^{\circ}\right)$ & $99.28(4)$ & $98.92(4)$ & $98.63(5)$ \\
\hline $\mathrm{V}(\AA 3)$ & $770.6(3)$ & $773,5(3)$ & $776,1(5)$ \\
\hline
\end{tabular}


A detailed study was performed (with more temperatures datas) using conventional X-ray source [6], but data with more resolution need to be performed (using synchrotron radiation sources) in order to really match the properties of this amino-acid with the structural changings. However, in our preliminary study, it is possible to observe the following results:

a. The lattice parameters $\mathrm{a}$ and $\mathrm{c}$ increase with the temperature increasing (Figure 4a-4d),

b. In the lattice parameter b we observe an anomaly around $200 \mathrm{~K}$,

c. The beta angle decreases with temperature as observed in the literature; and finally,

d. The general volume of the crystal increase with temperature (Figure 4e).

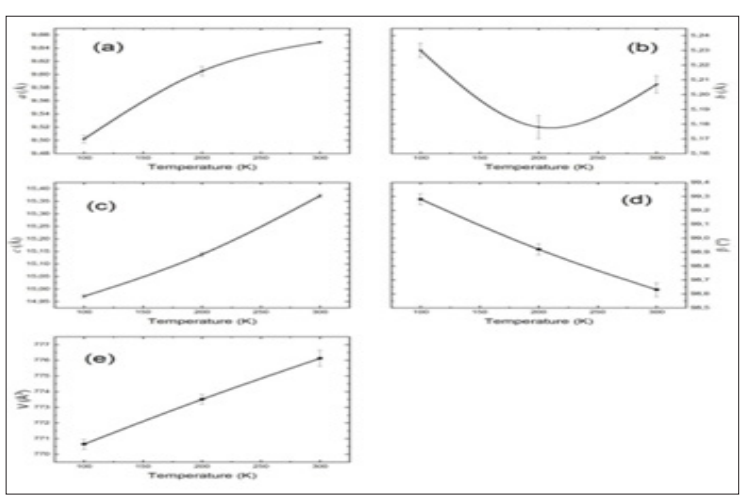

Figure 4: (a) lattice parameter a, (b) b, (c) c, (d) beta angle and (e) volume for 3 temperatures $(100 \mathrm{~K}, 200 \mathrm{~K}$ and 300 $\mathrm{K})$, as a result of Rietveld refinement of the X-ray and neutron data.

In order to confirm the anomalies around $200 \mathrm{~K}$, more measurements, with small step in temperature, are necessary to be performed. In addition, to have a complete view of the behavior of the light elements (as for instance, hydrogen) in the structure, a combined experiment using neutron powder diffraction is desirable.

\section{ISSN: 2574-1241}

DOI: 10.26717/BJSTR.2018.08.001643

Margareth KKD Franco. Biomed J Sci \& Tech Res

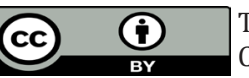

This work is licensed under Creative Commons Attribution 4.0 License

Submission Link: https://biomedres.us/submit-manuscript.php

\section{Conclusion}

The structural analysis of the X-ray diffraction patterns confirmed the existence of an anomaly around $200 \mathrm{~K}$, nevertheless a more detailed measurement in temperature still need to performed. In the preliminaries results obtain at XPD-LNLS combined with a neutron measurement at Aurora-IPEN, it is possible to observe the changing in the structure of the L-methionine. Further refinement and measurement are necessary to really characterize this phase transitions and the polymorphism behavior of this compound. It is clear that L-met is an excellent model system that can allow a better understanding of the intricate structure-property relation in small molecular arrangements.

\section{Acknowledgement}

The authors would like to thank Dr. VL. Mazzochi and Dr. C.B.R. Parente for performing Neutron Difraction experiments.

\section{References}

1. Van Brummelen R, Du Toit (2007) L-methionine as immune supportive supplement: a clinical evaluation. D Amino Acids 33(1): 157-163.

2. Moskovitz J (2005) Methionine Oxidation and Methionine Sulfoxide Reductases Biochimica ET Biophysica Acta-Proteins and Proteomics 1703(2): 213-219.

3. Fischer J, Lima JA, Freire PTC, Mello FEA, Havenith RWA, et al. (2013) Molecular flexibility and structural instabilities in crystalline L-methionineBiophysical Chemistry (180-181): 76-85.

4. Parente CBR, Mazzochi VL, Mestnik-Filho J, Mascarenhas YP, Berliner R (2010) Aurora-A high-resolution powder diffractometer installed on the IEA-R1 research reactor at IPEN-CNEN/SP. Nuclear Instruments and Methods in Physics Research A 622(3): 678-684.

5. Ferreria FF, Granado E, Carvalho W Jr, Kycia SW, Bruno D, et al. (2006) Journal Synchrotron Radiation 13: 46-53.

6. Rietveld H (1969) A profile refinement method for nuclear and magnetic structures. J Appl Crystallogr 2: 65-71.

7. Rodriguez-Carbajal J (1980) FULLPROF: a program for Rietveld refinement and pattern matching analysis, Abstract of the Stellite Meeting on Powder Diffraction of the XV Congres of the IUCr.

8. Coelho AA, evans J, Evans I, Kem A, Parsons S (2011) Powder Diffract 26: $22-25$.

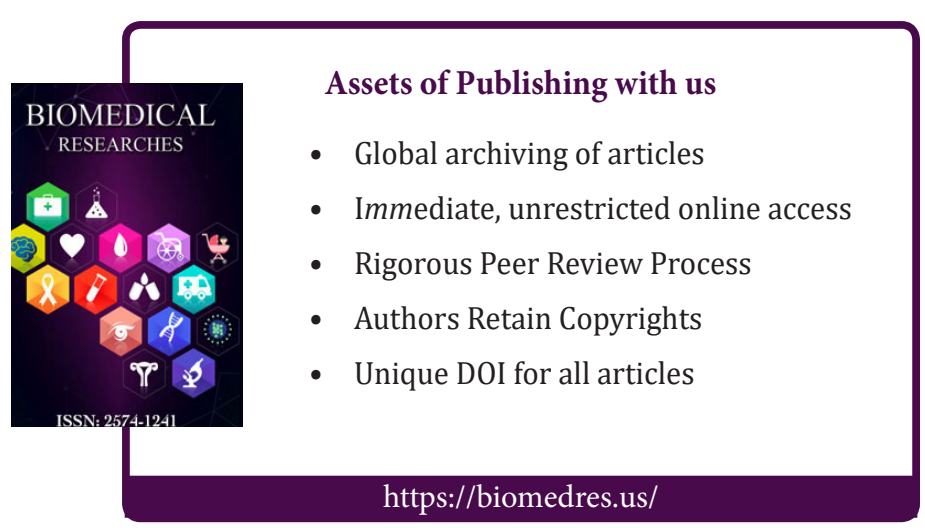

